TITLE:

\title{
Near-field evidence of local polarized emission centers in InGaN/GaN materials
}

$\operatorname{AUTHOR}(S)$ :

Micheletto, Ruggero; Allegrini, Maria; Kawakami, Yoichi

\section{CITATION:}

Micheletto, Ruggero ...[et al]. Near-field evidence of local polarized emission centers in InGaN/GaN materials. APPLIED PHYSICS LETTERS 2009, 95(21): 211904.

\section{ISSUE DATE:}

2009-11

URL:

http://hdl.handle.net/2433/109905

\section{RIGHT:}

Copyright 2009 American Institute of Physics. This article may be downloaded for personal use only. Any other use requires prior permission of the author and the American Institute of Physics. The following article appeared in APPLIED PHYSICS LETTERS 95, 211904 (2009) and may be found at

http://link.aip.org/link/APPLAB/v95/i21/p211904/s1 


\title{
Near-field evidence of local polarized emission centers in InGaN/GaN materials
}

\author{
Ruggero Micheletto, ${ }^{1, a)}$ Maria Allegrini, ${ }^{2}$ and Yoichi Kawakami ${ }^{3}$ \\ ${ }^{1}$ Nanoscience and Technology, International Graduate School of Art and Sciences, \\ Yokohama City University, Seto 22-2, Kanazawa-ku, Yokohama 236-0027, Japan \\ ${ }^{2}$ Department of Physics "E. Fermi," University of Pisa and CNISM, Largo Pontecorvo 3, 56127 Pisa, Italy \\ ${ }^{3}$ Department of Electronic Science, Graduate School of Engineering, Kyoto University, Nishigyo-ku, \\ Katsura, 615-8510 Kyoto, Japan
}

(Received 21 July 2009; accepted 28 October 2009; published online 24 November 2009)

\begin{abstract}
We study the optical polarization properties of confined structures in InGaN/GaN single quantum well devices. Using a near-field optical setup we investigated the photoluminescence maps with a polarization-modulation method. If the optical emissions have a preferred polarization orientation, our apparatus yields a signal that is proportional to the degree of polarization. We could demonstrate that within the quantum well there are localized submicrometer centers that emit strongly oriented light. This points toward the existence of quantum-dot like confined asymmetric domains hidden within the quantum well. (C) 2009 American Institute of Physics. [doi:10.1063/1.3265732]
\end{abstract}

Wide band solid state materials are used internationally in the effort to realize more efficient and more luminous optical devices as light emitting devices, lasers, and similar objects. Indium gallium nitrides (InGaN) are currently used for various applications in this field, for example, the creation of shorter wavelength devices for optical memory read/write applications ${ }^{1}$ or for illumination systems. $^{2}$

It has been noticed, ${ }^{1-3}$ that the optical emission of these samples is not uniform but is characterized by a granular structure with localized stronger centers. The nature of these confined higher emission domains is not clear; they present peculiar shapes, can show blinking, ${ }^{4}$ and often are heard to be associated with quantum dots or highly confined dotlike features in the crystal structure. These can be defects or impurities that somehow act as carriers traps in the quantum well. ${ }^{1,2}$ Many researchers suspect that these structures may have an inherent orientation or symmetry ${ }^{5}$ that can be observed optically by analyzing the polarization properties of the emission. In this letter we study for the first time an InGaN sample in high resolution with a very straightforward polarization-modulation technique.

The structure of a typical $\mathrm{GaN} / \mathrm{InGaN} / \mathrm{GaN}$ device is shown in the picture (Fig. 1). The active layer is a $3 \mathrm{~nm}$ quantum well. Indium concentration varies creating sometime areas of higher carrier recombination, known as intense luminous centers (ILCs). ${ }^{3}$

Our setup is a scanning near-field optical microscope (SNOM or NSOM). ${ }^{6}$ These devices are not refraction limited, they can reach resolution down to $50 \mathrm{~nm}$ or better. Standard measurements are not sensitive to polarization but opportunely modified system can detect rigorously the polarization characteristics of an emitter. ${ }^{7,8}$ Our main interest is to proof the presence of strongly polarized domains, while other properties of the polarization as phase, or other parameters are not relevant to us. For this reason we added to our SNOM apparatus a simplified polarization-modulation system able to detect the presence of polarization properties in a

${ }^{\text {a)} E l e c t r o n i c ~ m a i l: ~ r u g g e r o @ y o k o h a m a-c u . a c . j p . ~}$ photoluminescence pattern, straightforwardly, and with lowcost elements.

The system realizes simultaneous optical near-field and polarization properties mapping. It is based on a polarization-modulation technique (PM-SNOM) that was designed and theoretically modeled explicitly for application with optical materials analysis. ${ }^{9}$ The technique was first implemented to observe the polarization characteristics of diamond materials nanodislocations. ${ }^{10}$

We prove here that some InGaN confined emission centers do indeed emit light with strong polarization characteristics. We were able to discriminate luminescence intensity and determine the relative polarization degree of the optical emission. Optical emission is detected by the scanning nearfield optical microscope fitted with a nanoapertured optical probe. ${ }^{11-14}$ The probe-sample distance is in the order of few tenth of nanometer, it is monitored and kept constant using shear force method. ${ }^{15}$

The optical configuration ${ }^{9}$ consists of a $\lambda=442 \mathrm{~nm}$ $\mathrm{He}-\mathrm{Cd}$ laser (Kimmon, Ik series, $300 \mathrm{~mW}$ ) that is used as source, the light pass through a $\lambda / 4$ plate in order to obtain circular polarization; light is then coupled to the SNOM probe that induce locally the sample photoluminescence. We used double tapered probes with $200 \mathrm{~nm}$ nominal aperture, Cr-Gold coated (JASCO Corporation, Japan). These probes have a high-throughput of about $10^{-4}$, so about $30 \mu \mathrm{W}$ of (a)

\begin{tabular}{|c|}
\hline GaN $5 \mathrm{~nm}$ \\
\hline $\ln _{\mathrm{x}} \mathrm{Ga}_{1-\mathrm{x}_{\mathrm{x}}} \mathrm{Nnm}$ \\
\hline GaN $4 \mu \mathrm{m}$ \\
\hline Sapphire \\
\hline
\end{tabular}

(b)

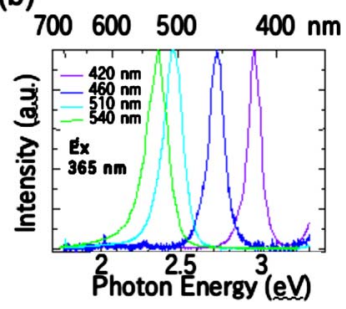

FIG. 1. (Color online) The samples we used are grown by metal-organic chemical-vapor deposition method on a sapphire substrate. The active layer is a $3 \mathrm{~nm}$ quantum well of indium doped gallium nitride. Depending on the indium concentration, the bulk material emits a spectrum of wavelength peaked at different values as shown in the right panel. 


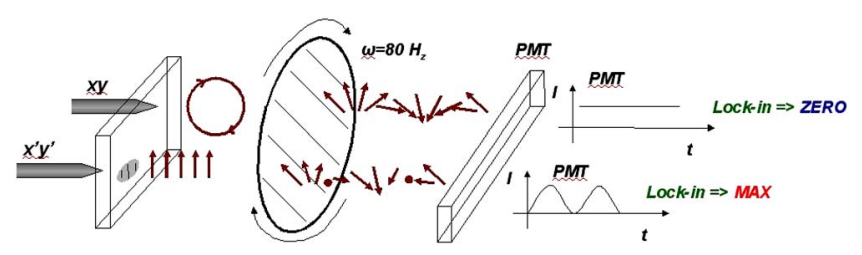

FIG. 2. (Color online) A schematic explanation of the detection mechanism: a quasicircular polarized light is launched on the sample by a local near-field probe. The PL light is filtered by a linear filter (Polaroid) that is put in between the sample and the photodetector (a photomultiplier indicated as PMT in the picture). If the photoluminescence preserves polarization properties, the emission will be still circular, and the signal at the PMT will be constant in value. If this condition is not true, the light will be filtered in amplitude by the Polaroid at the frequency of rotation of the Polaroid. A lock-in amplifier synchronized to this frequency will amplify all the relevant modulations.

optical power is finally available for inducing fluorescence on the InGaN surface. The samples used here have an emission peak centered on $540 \mathrm{~nm}$, so we used a $500 \mathrm{~nm}$ colored filter to cut-off the excitation signal. Fluorescence light coming off the sample is passed through a Polaroid mounted on a rotating motor (a modified light-chopper actuator) at a frequency of about $\omega_{r}=70 \mathrm{~Hz}$. The intensity values detected by the photomultiplier (PMT) are fed to a lock-in amplifier that is tuned with the Polaroid rotating frequency $\omega_{r}$. A personal computer records the two signals simultaneously and generates two maps, the near-field intensity map from the PMT, called SNOM image, and the map of the data from the lock-in that we call hereafter PM-SNOM map.

The physical meaning of a SNOM map is well known, ${ }^{11}$ it represents the intensity of the evanescent optical field in the vicinity of the sample surface. A PM-SNOM map ${ }^{7,9,16}$ is instead the results of the lock-in filtering, so it is an information related to the local polarization properties of the sample. If photoluminescence has not particular polarization, the intensity at the phototube will be constant in time. Instead, where there is local dichroism, signal will be oscillating at $\omega_{r}$. The amplitude of the oscillation represent how elliptic is the beam and it is measured directly at the phototube (PMT). A zero to one type of signal at PMT represents a linear polarization whereas a 0.9 to one signal represent an elliptic beam with $10 \%$ ratio between the two axis. The sample is scanned on the $x y$-plane, only the position dependent polarization properties are relevant in these maps. Polarization changes introduced by the birefringence of the tip or other tip-introduced spurious phenomena are not relevant because they are not position dependent and result in a mere elliptical excitation light, or a background offset in the PM-SNOM image. Naturally, the unavoidable polarization distortion introduced by the tip should be not too big. In fact, for our system to work and exhibit polarization contrast, the excitation beam polarization should cover all the angular spectrum. If this is not true measurements would not be possible, we could verify that the JASCO probes used conserve polarization well enough to have a reasonable quasicircular excitation beam and good polarization contrast in our maps.

We modeled and simulated the whole optical cascade, considering also the effects of real instrumentations as lock-in and photon counter. ${ }^{9}$ If the $x y$ index indicates all position-dependent parameters, the signal mapped by the lock-in $R_{x y}$ is proportional to (a)

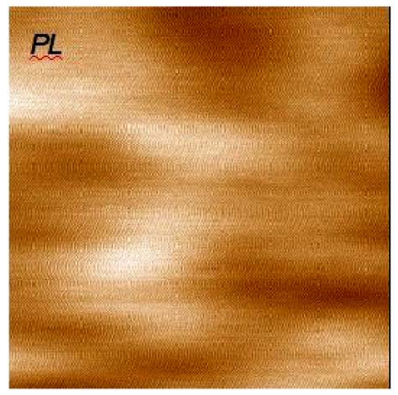

(b)

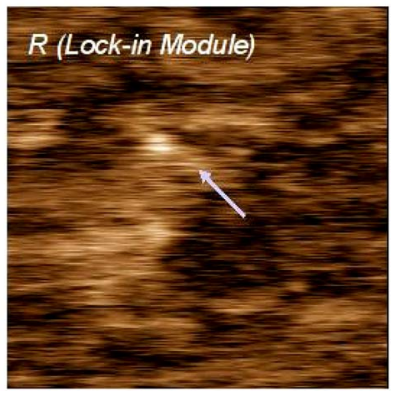

FIG. 3. (Color online) (a) The surface mapping of a $\lambda=540 \mathrm{~nm} \mathrm{InGaN}$ sample taken in near-field (color filter $\lambda=500 \mathrm{~nm}$ ). The picture in the panel (b) shows the lock-in signal. Features invisible in (a) emerge, indicating the presence of confined polarization active regions associable to localized domains within the crystal lattice (quantum dots). The two maps are recorded simultaneously. Image size is $7.5 \times 7.5 \mu \mathrm{m}$ composed of $256 \times 256$ pixels.

$$
R_{x y}=k_{x y} \frac{\pi \rho_{x y}}{2 \omega_{r}}
$$

where $k$ represents the local transparency of the sample, whereas $\rho$ is the actual polarization map. This parameter [Eq. (1)] is null where there are not polarization changing properties and it is linearly proportional to them. Since our sample on small scales can be considered of constant transparency, $k_{x y}$ is a constant for all points $x y$. Because $\omega_{r}$ is another constant, the lock-in $R_{x y}$ map is proportional to the polarization properties of the sample. See Fig. 2 for a simplified visual scheme of the principle of operation of the setup. Simultaneous maps are recorded and shown in Fig. 3; the near field optic emission (a) and the lock-in map (b). In (a) there are visible areas of higher optical intensity caused by the typical Indium concentration variations.,

The lock-in generated map (b) reveals a confined area of strong polarization that is not detectable in the standard SNOM map (a). This indicates the relevance of the methodology to put forward quantum dot like local polarizationchanging domains that could not be detected normally. A residual background position-independent intensity is due to spurious polarization introduced by the optical probe dichroism. Also, all the features in (b) do not have corresponding counterpart in (a), demonstrating that there are not artifacts due to cross talk. In Fig. 4 we show another example of quantum-dot like features revealed by the tests, panel (b) shows that the feature has strong polarization properties. (a)

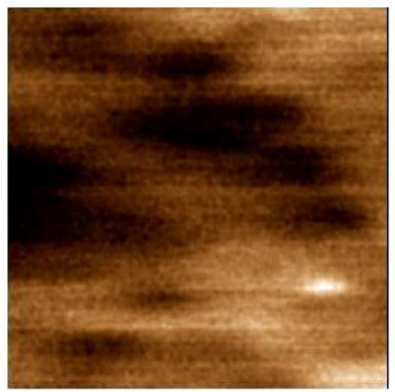

(b)

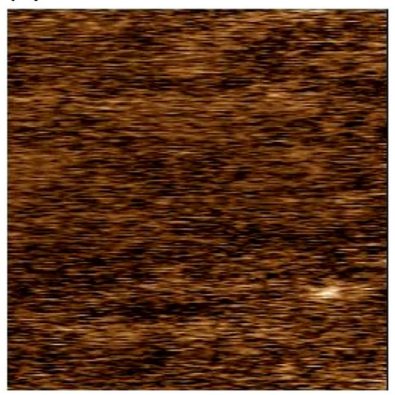

FIG. 4. (Color online) A confined submicrometer emission center seen in the near-field photoluminescence map (a) and the simultaneously taken polarization properties map (b). Image size is $3.75 \times 3.75 \mu \mathrm{m}$ composed of $256 \times 256$ pixels. 
Measurements were done with a scanning speed of $36 \mathrm{~ms}$ per pixel, integrating with the lock-in with a time constant $\tau=10 \mathrm{msec}$. Total scan time was of about $40 \mathrm{~min}$. A residual horizontal elongation of features is an instrumental artifact due to the slow integration time compared to the scan speed.

The presence of highly confined carrier traps can induce the local ILCs mapped. The fact that some of them have inherent polarization properties can be related to the geometrical symmetry of the confinement. If there are asymmetries or preferred orientation in the trap, we may observe the local polarization revealed by our tests. $5,17,18$

In other tests we do not show here for reason of space, it was noticed that not all the ILCs do have polarization properties, suggesting that asymmetrical strain is not a general characteristics of them but emerges randomly without a recognizable pattern.

This technique provide a high-resolution methodology for the general investigation of any optical emitting material to realize better understanding on the optical emission fundamental mechanism and possibly pave the way for the realization of better and more efficient devices.

We would like to thank Akio Kaneta, Kazunobu Kojima, and Dr. Tatsuo Nakagawa of Unisoku Ldt for helpful discussions and technical support with the SNOM apparatus. This study received financial contributions from the Strategic Research Project K20019 of Yokohama City University and from the executive program of scientific cooperation between Italy and Japan (Project 31).

${ }^{1}$ T. Mukai and S. Nakamura, Jpn. J. Appl. Phys., Part 1 38, 5735 (1999).

${ }^{2}$ S. Nakamura, Thin Solid Films 343, 345 (1999).

${ }^{3}$ R. Micheletto, N. Yoshimatsu, A. Kaneta, Y. Kawakami, and S. Fujita, Appl. Surf. Sci. 229, 338 (2004).

${ }^{4}$ R. Micheletto, M. Abiko, A. Kaneta, and Y. Kawakami, Appl. Phys. Lett. 88, 061118 (2006).

${ }^{5}$ O. Stier, M. Grundmann, and D. Bimberg, Phys. Rev. B 59, 5688 (1999).

${ }^{6}$ E. Betzig and J. K. Trautman, Science 257, 189 (1992).

${ }^{7}$ A. Ambrosio, M. Alderighi, M. Labardi, L. Pardi, F. Fuso, M. Allegrini, S. Nannizzi, A. Pucci, and G. Ruggeri, Nanotechnology 15, S270 (2004).

${ }^{8}$ T. Lacoste, T. Huser, R. Prioli, and H. Heinzelmann, Ultramicroscopy 71, 333 (1998).

${ }^{9}$ R. Micheletto, M. Allegrini, and Y. Kawakami, J. Opt. A, Pure Appl. Opt. 9, 431 (2007).

${ }^{10}$ R. Micheletto, Y. Kawakami, C. Manfredotti, Y. Garino, and M. Allegrini, Appl. Phys. Lett. 89, 121125 (2006).

${ }^{11}$ E. Betzig, J. Trautman, T. Harris, J. Weiner, and R. Kostelak, Science 251, 1468 (1991).

${ }^{12}$ T. Pangaribuan, K. Yamada, S. Jiang, H. Ohsawa, and M. Ohtsu, Jpn. J. Appl. Phys., Part 2 31, L1302 (1992).

${ }^{13}$ P. Lambelet, A. Sayah, M. Pfeffer, C. Philipona, and F. Marquis-Weible, Appl. Opt. 37, 7289 (1998).

${ }^{14}$ R. Stockle, C. Fokas, V. Deckert, R. Zenobi, B. Sick, B. Hecht, and U. P. Wild, Appl. Phys. Lett. 75, 160 (1999).

${ }^{15}$ W. Gohde, J. Tittel, T. Basche, C. Brauchle, U. C. Fisher, and H. Fuchs, Rev. Sci. Instrum. 68, 2466 (1997).

${ }^{16}$ L. Ramoino, M. Labardi, N. Maghelli, L. Pardi, M. Allegrini, and S. Patane, Rev. Sci. Instrum. 73, 2051 (2002).

${ }^{17}$ S. Chichibu, T. Azuhata, T. Sota, and S. Nakamura, Appl. Phys. Lett. 69, 4188 (1996).

${ }^{18}$ A. Shikanai, T. Azuhata, T. Sota, S. Chichibu, A. Kuramata, K. Horino, and S. Nakamura, J. Appl. Phys. 81, 417 (1997). 